\title{
Immunohistochemistry and clinical value of sirtuin 2 in non- metastasized non-small cell lung cancer
}

\author{
Cai-Xia Gao ${ }^{1}$, Bin Chen ${ }^{2}$, Hui-Kang Xie ${ }^{1}$, Chao-Nan Han ${ }^{2}$, Jie Luo ${ }^{2}$ \\ ${ }^{1}$ Department of Pathology, ${ }^{2}$ Department of Oncology, Shanghai Pulmonary Hospital, Tongii University School of Medicine, Shanghai 200433, \\ China \\ Contributions: (I) Conception and design: CX Gao, J Luo; (II) Administrative support: HK Xie; (III) Provision of study materials or patients: CX Gao, \\ J Luo; (IV) Collection and assembly of data: J Luo, CN Han; (V) Data analysis and interpretation: CX Gao, J Luo, B Chen, HK Xie; (VI) Manuscript \\ writing: All authors; (VII) Final approval of manuscript: All authors. \\ Correspondence to: Jie Luo. Department of Oncology, Shanghai Pulmonary Hospital, Tongji University School of Medicine, 507 Zhengmin Road, \\ Shanghai 200433, China. Email: 1j_1342@163.com.
}

Background: This study aimed to define whether sirtuin 2 (SIRT2) expression levels are related to the prognosis of non-small cell lung cancer (NSCLC) patients.

Methods: A survival analysis was carried out using the Kaplan-Meier (KM) plotter database. Immunohistochemical staining was performed and KM's method was used to estimate the survival rates for SIRT2 expression in 72 clinical samples.

Results: A survival analysis of 1,926 NSCLC patients showed that patients with low SIRT2 expression levels had significantly longer overall survival (OS) than those with high SIRT2 expression levels $(\mathrm{P}=0.0077$; HR =1.19). In 72 non-metastasized NSCLC tissues, the positive rate of SIRT2 expression was $90.3 \%$ (65/72), among which, the positive expression rates of squamous cell carcinoma (SCC) and adenocarcinoma (ADC) were 96.4\% (27/28) and 85.4\% (35/41), respectively. Survival analysis showed that patients with low SIRT2 expression levels had significantly longer median survival time (MST) than those with high SIRT2 expression levels (15.0 versus 14.0 months, $\mathrm{P}=0.029)$. Furthermore, the results of subgroup analysis demonstrated patients with low SIRT2 expression levels had significantly longer survival time in ADC group (15.0 versus 12.0 months, $\mathrm{P}=0.022$ ), but there wasn't significant difference in SCC group (15.0 versus 14.0 months, $\mathrm{P}=0.932$ ). A multivariate Cox proportional hazards model, which included gender, age, TNM stage, differentiation and SIRT2 expression, showed that SIRT2 expression was an independent factor related to prognosis [HR $=1.903,95 \%$ confidence interval ( $95 \% \mathrm{CI}): 1.085-3.339, \mathrm{P}=0.025]$.

Conclusions: SIRT2 expression levels were significantly related to the survival time of patients with lung ADC but not SCC. Our study indicated SIRT2 was perhaps a specific prognostic biomarker for nonmetastasized lung ADC.

Keywords: Non-small cell lung cancer (NSCLC); sirtuin 2 (SIRT2); immunohistochemistry; prognosis

Submitted Feb 15, 2019. Accepted for publication Aug 06, 2019.

doi: $10.21037 /$ jtd.2019.08.102

View this article at: http://dx.doi.org/10.21037/jtd.2019.08.102

\section{Introduction}

Despite recent progress in detection and therapy, lung cancer is still the most prevalent malignant tumour and the leading cause of cancer-related mortality worldwide (1). Non-small cell lung cancer (NSCLC) comprises approximately $85 \%$ of all primary lung cancer cases (2). Nearly all of these patients have unresectable advanced disease with lymph nodes and/or visceral metastases at the time of diagnosis, which indicates poor curative effects and prognosis. Thus, the identification of new prognostic biomarkers for NSCLC is needed to improve postoperative treatment strategies. 
Table 1 Characteristics of 72 NSCLC patients

\begin{tabular}{|c|c|c|c|}
\hline $\begin{array}{l}\text { Characteristics } \\
(n=72)\end{array}$ & $\begin{array}{l}\text { ADC, } \\
\mathrm{n}(\%)\end{array}$ & $\begin{array}{l}\text { SCC, } \\
\text { n (\%) }\end{array}$ & $\begin{array}{c}\text { Others, } \\
\text { n (\%) }\end{array}$ \\
\hline \multicolumn{4}{|l|}{ Age/years } \\
\hline$\leq 60$ & $20(27.8)$ & $9(12.5)$ & $1(1.4)$ \\
\hline$>60$ & $21(29.2)$ & $19(26.4)$ & $2(2.8)$ \\
\hline \multicolumn{4}{|l|}{ Gender } \\
\hline Male & $25(34.7)$ & $19(26.4)$ & $2(2.8)$ \\
\hline Female & $16(22.2)$ & $9(12.5)$ & $1(1.4)$ \\
\hline \multicolumn{4}{|l|}{ Stage } \\
\hline 1 & $11(15.3)$ & $7(9.7)$ & $1(1.4)$ \\
\hline II & $19(26.4)$ & 10 (13.9) & $2(2.8)$ \\
\hline III & $11(15.3)$ & $11(15.3)$ & $0(0)$ \\
\hline \multicolumn{4}{|l|}{ Differentiation } \\
\hline Poorly & 10 (13.9) & $7(9.7)$ & $2(2.8)$ \\
\hline Moderately & $31(43.1)$ & $21(29.2)$ & $1(1.4)$ \\
\hline
\end{tabular}

Sirtuins (SIRTs), silent information regulator 2 (Sir2)related enzymes, are a class of proteins that possess a nicotinamide adenine dinucleotide (NAD+)-dependent class III histone deacetylase. SIRTs can deacetylate a variety of histones and non-histones, including NF- $\mathrm{BB}, \mathrm{p} 53$, and others. They are also key regulators of a wide variety of cellular and physiological processes such as cell proliferation, differentiation, DNA damage and the stress response, genome stability, metabolism, and tumourigenesis (3).

Mammals have seven SIRTs, namely, SIRT1-7. All mammalian SIRTs, except for SIRT5 have been reported to be involved in tumourigenesis. Among them, SIRT2 has drawn an increasing amount of attention due to its dual function in tumourigenesis and tumour development.

The role of SIRT2 in cancer is controversial because it may have a dual role as a tumour promoter or suppressor, depending on the tumour type. One genetic study indicated that ageing SIRT2-knockout mice show increased tumour incidence compared with wild-type controls, suggesting a potential role for SIRT2 as a tumour suppressor (4). In contrast, SIRT2 was also observed to have tumourpromoting activity in several studies (5-8). Moreover, several SIRT2 inhibitors have also been reported to have anticancer effects (9-13), indicating that SIRT2 may become the new target of anti-tumour therapy.

To address the possible role of SIRT2 in NSCLC, the relationship between SIRT2 expression and NSCLC patient survival was analysed using both the KaplanMeier (KM) plotter database (14) and 72 newly collected NSCLC surgical specimens. In addition, the influence of SIRT2 on both lung adenocarcinoma (ADC) and squamous cell carcinoma (SCC) patients' prognoses was estimated accordingly.

\section{Methods}

\section{Patients and samples}

Our research is a retrospective study. The study cases were selected from stage I to III NSCLC patients who underwent radical surgery in the Department of Thoracic Surgery at Shanghai Pulmonary Hospital between June 2011 and October 2015. The selection criteria for NSCLC patients consisted of several parameters: (I) patients with NSCLC confirmed by postoperative pathological examination; (II) patients between 18 and 80 years old; (III) patients with complete follow-up data and death were confirmed at the last follow-up; and (IV) patients with adequate paraffinembedded specimens in the Department of Pathology at Shanghai Pulmonary Hospital that were allowed to be used for scientific research.

A total of 72 cases (26 women and 46 men) who met the inclusion criteria were included in the study. Their ages ranged from 36 to 78 years, and the median age was 63 years. The pathological types consisted of 41 cases of ADC, 28 cases of SCC, and three others (including two cases of large cell neuroendocrine carcinoma and one case of adenosquamous carcinoma). The degree of tumour differentiation included 53 and 19 cases that were moderately and poorly differentiated, respectively. Most cases were in the early/middle stages, with 19,31 , and 22 cases in phases I, II, and III, respectively. Staging was based on the seventh edition of the Tumor Node Metastasis (TNM) staging system of The International Association for the Study of Lung Cancer (IASLC). Overall survival (OS) was defined as the time from diagnosis to death. Table 1 summarizes the clinical and pathological characteristics of the 72 cases.

Ethical approval for the current study was obtained from Shanghai Pulmonary Hospital (Shanghai, China). All patients provided signed informed consent for their tissues to be used for scientific research. 


\section{Antibodies and reagents}

The rabbit monoclonal antibody against human SIRT2 (ab211033) was purchased from Abcam, Inc. (Burlingame, CA, USA). Antigen retrieval solution (K8004) and a REAL EnVision kit (K5007) were purchased from DAKO (Dako Corp., Glostrup, Denmark).

\section{Immunobistochemical staining}

Immunohistochemical staining was performed on formalinfixed and paraffin-embedded $4-\mu m$-thick histological tissue microarray sections. The sections were deparaffinized and rehydrated in a xylene and alcohol bath solution. Antigen unmasking was performed by pretreatment of the slides in $0.01 \mathrm{M}$ citrate buffer $(\mathrm{pH} 6.0)$ at $98{ }^{\circ} \mathrm{C}$ for 5 min using a microwave oven. The slides were then treated with $3 \%$ hydrogen peroxidase in methanol for $10 \mathrm{~min}$ followed by SIRT2 (1:1,000 DAKO) application. The slides were then washed in phosphate-buffered saline and examined with the REAL EnVision kit (K5007). All slides were counterstained with haematoxylin. For negative controls, the isotypematched monoclonal control antibody (mAb) was used. The immunohistochemical staining in these specimens was visualized under an Olympus CX31 microscope (Olympus, Center Valley, PA, USA). Cells with tan particles in the cytoplasm were considered SIRT2 protein-positive. The percentage of positively stained cells was calculated by counting the number of positively stained cells among the total number of cells from at least five randomly selected horizons for each section $(\times 400)$. Tumours with $>75 \%$ positively stained cells were considered strong positive (+++) for SIRT2 expression; tumours with between $25 \%$ and $75 \%$ positively stained cells were considered moderately positive (++); tumours with $<25 \%$ positively stained cells were considered weakly positive (+); and tumours without positively stained cells were considered negative (-).

\section{Statistical analysis}

All statistical analyses were performed using the statistical program SPSS version 17.0 (SPSS, Inc., Chicago, IL, USA). Data are presented as percentages, medians, and 95\% CI. KM's method was used to estimate the survival rates for SIRT2 expression. Univariate analysis of OS was tested by log-rank statistics. Then we enter the variables which came out to be significant in the univariate analysis $(\mathrm{P}<0.05$ as significant) into the regression model using forward step- wise selection. And the probability for entry and remove are $\leq 0.05$ and $\geq 0.10$, respectively. All tests were two-tailed, and results with $\mathrm{P}$ values $<0.05$ were considered statistically significant.

\section{Results}

\section{SIRT2 expression in relation to prognosis}

To evaluate whether SIRT2 expression levels can predict the prognosis of NSCLC patients, an OS analysis was performed in 1,926 NSCLC patients using the KM plotter database. OS analysis of 1,926 NSCLC patients showed that patients with low SIRT2 expression levels had significantly longer OS than those with high SIRT2 expression levels $(\mathrm{P}=0.0077 ; \mathrm{HR}=1.19)$ (Figure 1A). In 720 lung ADC patients, the survival analysis showed that patients with low SIRT2 expression levels had significantly longer OS than those with high SIRT2 expression levels $(\mathrm{P}=0.00013$; $\mathrm{HR}=1.6$ ) (Figure 1B). In 542 SCC patients, survival analysis showed that patients with low SIRT2 expression levels was comparable to that with high SIRT2 expression levels $(\mathrm{P}=0.19 ; \mathrm{HR}=0.84)$ (Figure 1C).

To validate this finding at the protein level, SIRT2 expression was analysed via immunohistochemical staining in 72 tumour samples from NSCLC patients (Figure 2A). The KM analysis showed that patients with low (negative or weakly positive) SIRT2 expression levels had significantly longer OS than those with high (moderately or strongly positive) SIRT2 expression levels (15.0 versus 14.0 months, $\mathrm{P}=0.029$ ) (Figure 2B).

In 41 lung ADC patients, the OS analysis showed that patients with low SIRT2 expression levels had significantly longer OS than those with high SIRT2 expression levels (15.0 versus 12.0 months, $\mathrm{P}=0.022$ ) (Figure $2 C$ ). In $28 \mathrm{SCC}$ patients, the survival analysis showed that the OS of patients with low SIRT2 expression levels was comparable to that of patients with high SIRT2 expression levels (15.0 versus 14.0 months, $\mathrm{P}=0.932$ ) (Figure 2D).

\section{The potential prognostic value of SIRT2 in NSCLC}

The correlation between the OS of NSCLC patients and clinicopathological characteristics was explored using a univariate analysis. As shown in Table 2, there was a significant association between the degree of tumour differentiation $(\mathrm{P}=0.003)$ and SIRT2 expression levels $(\mathrm{P}=0.029)$. To further evaluate the potential of SIRT2 

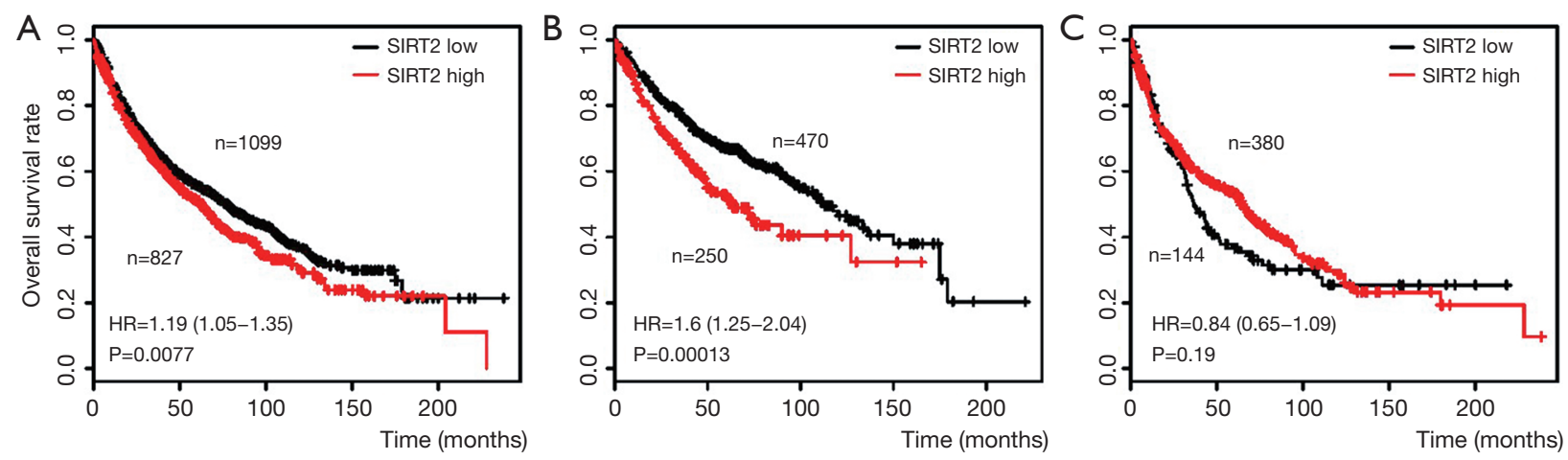

Figure 1 Transcription level of SIRT2 associated with prognosis in human lung adenocarcinoma. Kaplan-Meier curves of overall survival of NSCLC patients $(\mathrm{n}=1,926)(\mathrm{A})$, lung adenocarcinoma $(\mathrm{n}=720)(\mathrm{B})$ and squamous cell lung carcinoma (n=524) (C), stratified by SIRT2 expression level. Data was obtained from http://www.kmplot.com.
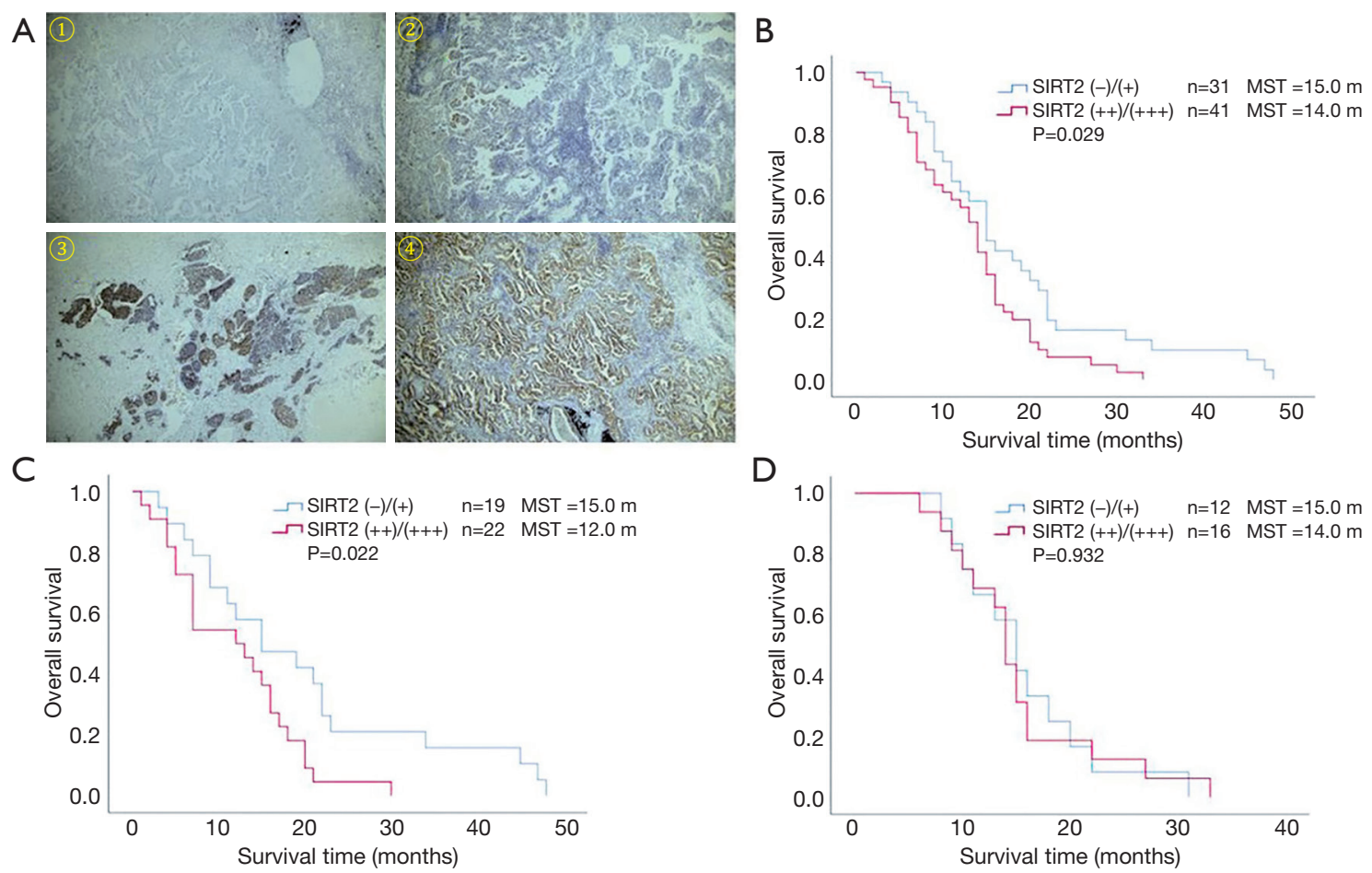

Figure 2 Protein level of SIRT2 associated with prognosis in human lung adenocarcinoma. (A) Representative image of immunohistochemical staining with an anti-SIRT2 antibody. (1), Negative (-): no positive cells; (2), weak positive (+): positive cell rate $<25 \%$; (3), moderate positive (++): $25-75 \%$ positive cell rate; (4) strong positive (+++): positive cell rate $>75 \%$ (400× in magnification). Kaplan-Meier curves of overall survival of NSCLC patients $(n=72)(B)$, lung adenocarcinoma $(n=41)(C)$, and squamous cell lung carcinoma $(\mathrm{n}=28)(\mathrm{D})$.

expression as a prognostic biomarker, a multivariate Cox proportional hazards model, which included gender, age, TNM stage, differentiation and SIRT2 expression level, for OS was carried out. As shown in Table 3, the OS of NSCLC patients correlated with SIRT2 expression levels $(\mathrm{P}=0.025)$ but not others, indicating that the SIRT2 expression level is an independent factor for prognosis. Negative or weak SIRT2 expression levels was associated with a better 
Table 2 Univariate analysis of overall survival

\begin{tabular}{|c|c|c|c|c|}
\hline Factor & $\begin{array}{l}\text { Case } \\
\text { (n) }\end{array}$ & $\begin{array}{l}\text { Median survival } \\
\text { time (month) }\end{array}$ & $95 \% \mathrm{Cl}$ & $P$ \\
\hline Gender & & & & 0.178 \\
\hline Male & 46 & 13 & $10.151-15.849$ & \\
\hline Female & 26 & 16 & $12.014-19.986$ & \\
\hline Age/years & & & & 0.075 \\
\hline$\leq 60$ & 30 & 15 & $12.316-17.684$ & \\
\hline$>60$ & 42 & 13 & $10.883-15.117$ & \\
\hline Pathologic types & & & & 0.716 \\
\hline $\mathrm{Ad}$ & 41 & 14 & $10.415-17.585$ & \\
\hline $\mathrm{Sq}$ & 28 & 14 & $12.518-15.482$ & \\
\hline Others & 3 & 9 & $4.199-13.801$ & \\
\hline TNM stage & & & & 0.204 \\
\hline 1 & 19 & 14 & $9.734-18.266$ & \\
\hline II & 31 & 15 & $12.722-17.278$ & \\
\hline III & 22 & 12 & $8.060-15.940$ & \\
\hline Differentiation & & & & 0.003 \\
\hline Poorly & 19 & 11 & 7.801-14.199 & \\
\hline Moderately & 53 & 15 & $13.057-16.943$ & \\
\hline SIRT2 level & & & & 0.029 \\
\hline$(-) /(+)$ & 31 & 15 & $11.742-18.258$ & \\
\hline$(++) /(+++)$ & 41 & 14 & $11.939-16.061$ & \\
\hline
\end{tabular}

+++ , strong positive, tumours with $>75 \%$ positively stained cells; ++, moderately positive, tumours with between $25 \%$ and $75 \%$ positively stained cells; + , weakly positive, tumours with $<25 \%$ positively stained cells; -, negative, tumours without positively stained cells. Ad, adenocarcinoma; Sq, squamous.

Table 3 Multivariate Cox proportional hazards model for overall survival

\begin{tabular}{lccc}
\hline Variables & HR & $95 \% \mathrm{Cl}$ & $\mathrm{P}$ \\
\hline Gender & 0.635 & $0.384-1.050$ & 0.077 \\
Age & 1.405 & $0.844-2.340$ & 0.191 \\
TNM stage & 1.356 & $0.916-2.008$ & 0.128 \\
Differentiation & 0.555 & $0.302-1.019$ & 0.057 \\
SIRT2 level & 1.903 & $1.085-3.339$ & 0.025 \\
\hline
\end{tabular}

prognosis $(\mathrm{HR}=1.903 ; 95 \% \mathrm{CI}: 1.085-3.339 ; \mathrm{P}=0.025)$.

\section{Discussion}

As a member of the SIRT family of proteins, SIRT2 is a type of NAD+-dependent deacetylase. It is widely expressed in different kinds of tissues and participates in normal physiological processes in addition to pathological cell processes (15). SIRT2 regulates several cell functions by mediating histone deacetylation, including genome stability, DNA damage repair, cell cycle progression, cellular oxidative stress, and inflammatory responses (16-18). An increasing number of studies in recent years have focused on SIRTs, particularly SIRT1, which is the most extensively studied SIRT; less is known about SIRT2. Mammalian SIRTs have been reported to be involved in tumourigenesis. However, the role(s) of SIRTs in cancer appears to be complex, and SIRTs may contribute to either tumour promotion or suppression. Their putative roles as either tumour suppressors or promoters remain to be validated.

Similar to SIRT1, increasing evidence has shown that SIRT2 has both tumour suppressor and promoter functions; however, the exact functions and mechanism of SIRT2 in tumourigenesis remain unclear. Kim found that SIRT2-knockout mice developed gender-specific tumourigenesis with females primarily developing mammary tumours, whereas males developed more hepatocellular carcinoma (4). They demonstrated that SIRT2 deficiency caused a reduction in the activity of the anaphase-promoting complex, thus causing an increase in the levels of mitotic regulators, including Aurora-A and Aurora-B, which direct centrosome amplification, aneuploidy, and mitotic cell death. These data suggest that SIRT2 functions as a tumour suppressor. On the other hand, other studies have suggested that SIRT2 acts as a tumour promoter (19).

McGlynn found that high levels of nuclear SIRT2 were associated with a shorter time to recurrence $(\mathrm{P}<0.001$, 5.47 versus 7.58 years $)$ and a shorter time to death $(\mathrm{P}=0.012$; 9.2 versus 12.6 years) in patients with grade $3 \mathrm{ER}$-ve breast tumours (20), showing the association between high SIRT2 nuclear expression and poor outcomes. Conversely, patients with ER+ve and grade 2 breast tumours whose tumours expressed low nuclear SIRT2 protein levels were more 
likely to relapse quicker ( $\mathrm{P}=0.047$; 9.2 versus 11.5 years), indicating that high SIRT2 protein levels are associated with a longer time to recurrence. These data suggest that SIRT2 has differential functions depending on the breast tumour grade. Therefore, it cannot be unilaterally classified as a tumour suppressor or promoter but rather can serve as both.

Until now, the role of SIRT2 in lung cancer has rarely been studied. Xu found that SIRT2 suppresses NSCLC growth by targeting Jumonji domain-containing protein 2A (JMJD2A), and the SIRT2 activator may serve as a candidate drug for NSCLC therapy (21). Conversely, another study by Hoffmann reported that SIRT2 inhibition caused an increase in $\mathrm{p} 53$ activation by causing a decrease in SIRT2-dependent p53 deacetylation (22); therefore, SIRT2 inhibitors may act as good therapeutic agents for NSCLC treatment. In addition, our previous study found that speckle-type POZ protein suppresses NSCLC growth by promoting SIRT2 degradation, indicating SIRT2's tumour promoter function in NSCLC (23).

Presently, most of the related SIRT2 studies are carried out at the cellular or animal level, but its role in NSCLC occurrence and development has seldom been implemented in research. In the present study, surgical specimens from NSCLC patients were collected to examine both SIRT2 gene and protein expression using immunohistochemical analysis. Furthermore, the correlation between SIRT2 expression and NSCLC patients' prognoses was evaluated to explore whether SIRT2 can be a prognostic factor for NSCLC and the way in which it works as a factor.

Our results show that the positive rate of SIRT2 expression in 72 cases of NSCLC tissues can be as high as $90.3 \%(65 / 72)$, among which, the positive expression rates of SCC and ADC were 96.4\% (27/28) and 85.4\% (35/41), respectively. Furthermore, the survival analysis showed that patients with negative or weakly positive SIRT2 expression levels had significantly longer OS rates than those with moderately or strongly positive levels $(\mathrm{P}=0.029 ; 15.0$ versus 14.0 months). Moreover, multivariate Cox proportional hazards analysis indicated that the SIRT2 expression level was an independent factor for prognosis, and negative or weak SIRT2 expression was associated with a better prognosis (HR =1.903; 95\% CI: 1.085-3.339; $\mathrm{P}=0.025$ ).

Grbesa et al. demonstrated that SIRT2 protein expression was significantly higher in lung primary tumours than in normal tissues, and high SIRT2 expression levels were associated with a poor prognosis in NSCLC patients; moreover, high SIRT2 expression was identified as an independent prognostic factor for shorter recurrence-free survival $(\mathrm{P}=0.007)$ (24). These results are consistent with our findings and further indicate that SIRT2 has a protumourigenic role in NSCLC.

\section{Conclusions}

In summary, our results suggest that non-metastasized lung ADC patients with low SIRT2 expression levels have a significantly better prognosis than those with high SIRT2 expression levels. SIRT2 was perhaps an independent prognostic biomarker in non-metastasized lung ADC but not SCC. This finding will help clinicians identify patients with poor prognosis and optimize treatment strategies to extend their survival time. It is noteworthy that a relatively modest number of NSCLC cases were examined in this study. Our research is just an explorative study. Large-scale studies will be needed to further verify our conclusions. Moreover, cell and animal models and clinical research should be used in future studies to clarify the exact role of SIRT2 in NSCLC occurrence and development. Taken together, our findings in this study provide a rationale for examining SIRT2 as a prognostic marker for and a therapeutic target in NSCLC patients.

\section{Acknowledgments}

None.

\section{Footnote}

Conflicts of Interest: The authors have no conflicts of interest to declare.

Ethical Statement: The authors are accountable for all aspects of the work in ensuring that questions related to the accuracy or integrity of any part of the work are appropriately investigated and resolved. We received ethical approval from the Ethics Committee of Shanghai Pulmonary Hospital to carry out this study (Permit Number K18-154). We also received informed consent for the participants to participate in the study.

\section{References}

1. Siegel R, Ma J, Zou Z, et al. Cancer statistics, 2014. CA Cancer J Clin 2014;64:9-29.

2. Chen W, Zhang S, Zou X. Estimation and projection of 
lung cancer incidence and mortality in China. Zhongguo Fei Ai Za Zhi 2010;13:488-93.

3. Yuan H, Su L, Chen WY. The emerging and diverse roles of sirtuins in cancer: a clinical perspective. Onco Targets Ther 2013;6:1399-416.

4. Kim HS, Vassilopoulos A, Wang RH, et al. SIRT2 maintains genome integrity and suppresses tumorigenesis through regulating APC/C activity. Cancer Cell 2011;20:487-99.

5. Chen J, Chan AW, To KF, et al. SIRT2 overexpression in hepatocellular carcinoma mediates epithelial to mesenchymal transition by protein kinase B/glycogen synthase kinase-3 $\beta / \beta$-catenin signaling. Hepatology 2013;57:2287-98.

6. Yang MH, Laurent G, Bause AS, et al. HDAC6 and SIRT2 regulate the acetylation state and oncogenic activity of mutant K-RAS. Mol Cancer Res 2013;11:1072-7.

7. Soung YH, Pruitt K, Chung J. Epigenetic silencing of ARRDC3 expression in basal-like breast cancer cells. Sci Rep 2014;4:3846.

8. Zhao D, Zou SW, Liu Y, et al. Lysine-5 acetylation negatively regulates lactate dehydrogenase $\mathrm{A}$ and is decreased in pancreatic cancer. Cancer Cell 2013;23:464-76.

9. Cheon MG, Kim W, Choi M, et al. AK-1, a specific SIRT2 inhibitor, induces cell cycle arrest by downregulating Snail in HCT116 human colon carcinoma cells. Cancer Lett 2015;356:637-45.

10. Kim WJ, Lee JW, Quan C, et al. Nicotinamide inhibits growth of carcinogen induced mouse bladder tumor and human bladder tumor xenograft through up-regulation of RUNX3 and p300. J Urol 2011;185:2366-75.

11. Jing H, Hu J, He B, et al. A SIRT2-selective inhibitor promotes c-myc oncoprotein degradation and exhibits broad anticancer activity. Cancer Cell 2016;29:297-310.

12. Heltweg B, Gatbonton T, Schuler AD, et al. Antitumor activity of a small-molecule inhibitor of human silent information regulator 2 enzymes. Cancer Res 2006;66:4368-77.

13. McCarthy AR, Sachweh MC, Higgins M, et al. Tenovin-D3, a novel small-molecule inhibitor of sirtuin

Cite this article as: Gao CX, Chen B, Xie HK, Han CN, Luo J. Immunohistochemistry and clinical value of sirtuin 2 in non-metastasized non-small cell lung cancer. J Thorac Dis 2019;11(9):3973-3979. doi: 10.21037/jtd.2019.08.102
SirT2, increases p21 (CDKN1A) expression in a p53independent manner. Mol Cancer Ther 2013;12:352-60.

14. Nagy Á, Lánczky A, Menyhárt O, et al. Validation of miRNA prognostic power in hepatocellular carcinoma using expression data of independent datasets. Sci Rep 2018;8:9227.

15. North BJ, Marshall BL, Borra MT, et al. The human Sir2 ortholog, SIRT2, is an NAD+-dependent tubulin deacetylase. Mol Cell 2003;11:437-44.

16. Serrano L, Martínez-Redondo P, Marazuela-Duque A, et al. The tumor suppressor SirT2 regulates cell cycle progression and genome stability by modulating the mitotic deposition of H4K20 methylation. Genes Dev 2013;27:639-53.

17. Nie H, Hong Y, Lu X, et al. SIRT2 mediates oxidative stress-induced apoptosis of differentiated PC12 cells. Neuroreport 2014;25:838-42.

18. Dryden SC, Nahhas FA, Nowak JE, et al. Role for human SIRT2 NAD-dependent deacetylase activity in control of mitotic exit in the cell cycle. Mol Cell Biol 2003;23:3173-85.

19. Liu PY, Xu N, Malyukova A, et al. The histone deacetylase SIRT2 stabilizes Myc oncoproteins. Cell Death Differ 2013;20:503-14.

20. McGlynn LM, Zino S, MacDonald AI, et al. SIRT2: tumour suppressor or tumour promoter in operable breast cancer? Eur J Cancer 2014;50:290-301.

21. Xu W, Jiang K, Shen M, et al. SIRT2 suppresses non-small cell lung cancer growth by targeting JMJD2A. Biol Chem 2015;396:929-36.

22. Hoffmann G, Breitenbücher F, Schuler M, et al. A novel sirtuin 2 (SIRT2) inhibitor with p53-dependent proapoptotic activity in non-small cell lung cancer. J Biol Chem 2014;289:5208-16.

23. Luo J, Bao YC, Ji XX, et al. SPOP promotes SIRT2 degradation and suppresses non-small cell lung cancer cell growth. Biochem Biophys Res Commun 2017;483:880-4.

24. Grbesa I, Pajares MJ, Martínez-Terroba E, et al. Expression of sirtuin 1 and 2 is associated with poor prognosis in non-small cell lung cancer patients. PLoS One 2015; 10:e0124670. 